This is an electronic reprint of the original article. This reprint may differ from the original in pagination and typographic detail.

Author(s): Ketterer, Christian; Rajala, Tapio

Title: $\quad$ Failure of Topological Rigidity Results for the Measure Contraction Property

Year: $\quad 2015$

Version:

Please cite the original version:

Ketterer, C., \& Rajala, T. (2015). Failure of Topological Rigidity Results for the Measure Contraction Property. Potential Analysis, 42(3), 645-655.

https://doi.org/10.1007/s11118-014-9450-5

All material supplied via JYX is protected by copyright and other intellectual property rights, and duplication or sale of all or part of any of the repository collections is not permitted, except that material may be duplicated by you for your research use or educational purposes in electronic or print form. You must obtain permission for any other use. Electronic or print copies may not be offered, whether for sale or otherwise to anyone who is not an authorised user. 


\title{
FAILURE OF TOPOLOGICAL RIGIDITY RESULTS FOR THE MEASURE CONTRACTION PROPERTY
}

\author{
CHRISTIAN KETTERER AND TAPIO RAJALA
}

\begin{abstract}
We give two examples of metric measure spaces satisfying the measure contraction property $\operatorname{MCP}(K, N)$ but having different topological dimensions at different regions of the space. The first one satisfies $\operatorname{MCP}(0,3)$ and contains a subset isometric to $\mathbb{R}$, but does not topologically split. The second space satisfies $\operatorname{MCP}(2,3)$ and has diameter $\pi$, which is the maximal possible diameter for a space satisfying $\operatorname{MCP}(N-1, N)$, but is not a topological spherical suspension. The latter example gives an answer to a question by Ohta.
\end{abstract}

\section{Introduction}

There are several generalizations of Ricci curvature lower bounds to metric measure spaces that use optimal mass transportation. The definitions that have received the most attention are the $\operatorname{CD}(K, N)$ definitions by Sturm [28, 29], and Lott and Villani [18]. In the definitions the first number $K \in \mathbb{R}$ always refers to a lower bound on the Ricci curvature and the second number $N \in[1, \infty]$ refers to an upper bound on the dimension of the space. A less restrictive version of Ricci curvature lower bounds, called the measure contraction property $\operatorname{MCP}(K, N)$ was studied by Sturm [29], and by Ohta [20, 21]. Recently also a more restrictive class of definitions called the Riemannian Ricci curvature lower bounds $\operatorname{RCD}^{*}(K, N)$ have been considered $[2,1,10,3]$.

Common to all these definitions is stability under the measured Gromov-Hausdorff convergence. However, with the $\operatorname{CD}(K, N)$ and $\operatorname{MCP}(K, N)$ definitions this stability has sometimes been sacrificed by assuming the space to be nonbranching. For the $\operatorname{RCD}^{*}(K, N)$ definitions such extra assumption seems unnecessary. This can be seen from the fact that such spaces are already essentially nonbranching $[27,13]$. For some time it was not clear if the nonbranching assumption was just a technical assumption that was made to simplify the proofs. This was the case for some of the results, like the local Poincaré inequalities $[26,25]$. However, it was recently shown by the second named author that in order to obtain a local-to-global property for $\operatorname{CD}(K, N)$ it is necessary to assume nonbranching [24]. Notice that, as our above discussion suggests, for the $\operatorname{RCD}^{*}(K, N)$ a nonbranching assumption is not needed for the local-to-global property [4, 13].

In this paper we further study the necessity of the nonbranching assumption in the theory of Ricci curvature lower bounds in metric measure spaces. This time we focus on

2000 Mathematics Subject Classification. Primary 53C23. Secondary 28A33, 49Q20.

Key words and phrases. Ricci curvature lower bounds, measure contraction property, splitting theorem, maximal diameter theorem, metric measure spaces, geodesics, nonbranching. 
geometric (or topological) rigidity results. More specifically, we look at two classical rigidity results from Riemannian geometry. The first result is the Cheeger-Gromoll splitting theorem [8] saying that a Riemannian manifold $\mathbf{M}$ with non-negative Ricci curvature containing an infinite line-segment is isometric to $\mathbb{R} \times \mathbf{N}$ where $\mathbf{N}$ is again a Riemannian manifold with non-negative Ricci curvature. The second rigidity result is the Cheng maximal diameter theorem [9] stating that an $n$-dimensional Riemannian manifold with Ricci curvature bounded from below by $n-1$ and with diameter $\pi$ is necessarily the standard sphere $\mathbb{S}^{n}$. Recall that, by the Bonnet-Meyers theorem, $\pi$ is the maximal possible diameter for an $n$-dimensional Riemannian manifold with Ricci curvature bounded from below by $n-1$.

Both of the above mentioned rigidity results have been proven in the abstract setting of $\mathrm{RCD}^{*}(K, N)$ spaces. The Cheeger-Gromoll splitting theorem was generalized to $\operatorname{RCD}(0, N)$ spaces by Gigli [11]. Using this generalization and by studying the metric cones over $\operatorname{RCD}^{*}(K, N)$ spaces the first named author recently generalized the Cheng maximal diameter theorem to $\operatorname{RCD}^{*}(K, N)$ spaces $[15]$.

Now that we know that the rigidity results hold for $\operatorname{RCD}^{*}(K, N)$ spaces it is natural to ask if they hold for the less restrictive definitions $\operatorname{CD}(K, N)$ and $\operatorname{MCP}(K, N)$. Since $\mathrm{CD}(K, N)$ spaces include nonRiemannian Finsler manifolds, it would be unreasonable to expect the Cheeger-Gromoll and Cheng theorems to hold in the same sharp form as for the $\operatorname{RCD}^{*}(K, N)$ spaces. However, Ohta has shown that topological versions of the rigidity results still hold in the Finsler setting. Indeed, he has proven a diffeomorphic splitting theorem for Finsler manifolds of nonnegative weighted Ricci curvature [22]. Ohta also proved in [21] that in nonbranching $\operatorname{MCP}(K, N)$ spaces a maximal diameter theorem holds in the following topological form.

Theorem 1.1 (Ohta [21]). Let $(X, \mathrm{~d}, \mu)$ be a compact metric measure space satisfying the $\operatorname{MCP}(N-1, N)$ property for some $N>1$ and assume that there exist $x_{N}, x_{S} \in X$ with $\mathrm{d}\left(x_{N}, x_{S}\right)=\pi$ such that

$$
\operatorname{Cut}\left(x_{N}\right) \backslash\left\{x_{S}\right\}=\operatorname{Cut}\left(x_{S}\right) \backslash\left\{x_{N}\right\}=\emptyset .
$$

Then there exists a topological measure space $(Y, \nu)$ such that $(X, \mu)$ is the spherical suspension of $(Y, \nu)$ as a topological measure space.

Here the cut locus $\operatorname{Cut}(x)$ of a point $x \in X$ is the set of points $z \in X$ such that there exist at least two distinct minimal geodesic between $x$ and $z$. Under the nonbranching assumption (1.1) is satisfied. In [21, 19] Ohta asked if the topological maximal diameter result holds for $\operatorname{MCP}(K, N)$ spaces without the nonbranching assumption. We show that this is not the case.

Theorem 1.2. There exists a compact geodesic metric measure space $(Y, \mathrm{~d}, \mathfrak{n})$ satisfying $\operatorname{MCP}(2,3)$ and $\operatorname{diam}(Y)=\pi$. Still the space is not a topological spherical suspension.

A slightly easier construction than the one showing the failure of the topological maximal diameter theorem shows that also topological splitting results fail for $\operatorname{MCP}(0, N)$ spaces. 
Theorem 1.3. There exists a complete geodesic metric measure space $(X, \mathrm{~d}, \mathfrak{m})$ satisfying $\operatorname{MCP}(0,3)$ and containing an isometrically embedded copy of $\mathbb{R}$. Still the space does not topologically split.

Still several questions remain open between the positive results and the counter-examples stated in Theorems 1.2 and 1.3. Two obvious questions are the following.

Question 1.4. Does a topological maximal diameter theorem hold in $\mathrm{CD}(K, N)$ spaces?

Question 1.5. Does a topological splitting theorem hold in $\operatorname{CD}(0, N)$ spaces?

Notice that there exist spaces satisfying $\operatorname{CD}(K, N)$ with positive $K$ that contain lots of branching geodesics [19], so Question 1.4 does not have an obvious answer via Theorem 1.1 .

These questions are also related to the local structure of spaces with different Ricci curvature lower bounds. It is still unknown even for $\operatorname{RCD}^{*}(K, N)$ spaces if the Hausdorff dimension or any other relevant dimension is constant almost everywhere. Perhaps the currently best positive result in this direction is that $\operatorname{RCD}^{*}(K, N)$ spaces have euclidean weak tangents at almost every point [12]. The examples of this paper show that the least restrictive definitions of Ricci curvature lower bounds discussed here - the $\operatorname{MCP}(K, N)$ conditions - do not imply that the Hausdorff (or topological) dimension of the space is almost everywhere constant.

\section{Preliminaries}

Before defining the spaces mentioned in Theorems 1.2 and 1.3 we recall some basics including the definition of $\operatorname{MCP}(K, N)$ and its connection to other definitions of Ricci curvature lower bounds in metric measure spaces.

First of all, by a geodesic $\gamma$ in a metric space $(X, \mathrm{~d})$ we mean a map $\gamma:[0,1] \rightarrow X$ satisfying

$$
\mathrm{d}(\gamma(t), \gamma(s))=|s-t| \mathrm{d}(\gamma(0), \gamma(1)) \quad \text { for all } s, t \in[0,1]
$$

The set of all geodesics in $(X, \mathrm{~d})$ is denoted by $\operatorname{Geo}(X)$. For $\gamma \in \operatorname{Geo}(X)$ we abbreviate $\gamma_{t}:=\gamma(t)$ for all $t \in[0,1]$. We also write length as $l(\gamma):=\mathrm{d}\left(\gamma_{0}, \gamma_{1}\right)$ for all $\gamma \in \operatorname{Geo}(X)$ and define the evaluation maps

$$
e_{t}: \operatorname{Geo}(X) \rightarrow X: \gamma \mapsto \gamma_{t} \quad \text { for all } t \in[0,1] .
$$

The measure contraction property $\operatorname{MCP}(K, N)$ is defined using the functions $\mathbf{s}_{K}$ with $K \in \mathbb{R}$. They are defined as

$$
\begin{aligned}
\mathbf{s}_{K}:[0, \pi / \sqrt{K}) \rightarrow \mathbb{R}: t \mapsto \frac{\sin (\sqrt{K} t)}{\sqrt{K}}, & \text { for } K>0, \\
\mathbf{s}_{K}:[0, \infty) \rightarrow \mathbb{R}: t \mapsto \frac{\sinh (\sqrt{-K} t)}{\sqrt{-K}}, & \text { for } K<0 \text { and } \\
\mathbf{s}_{K}:[0, \infty) \rightarrow \mathbb{R}: t \mapsto t, & \text { for } K=0 .
\end{aligned}
$$


Using these we define for all $t \in[0,1]$ and $d \geq 0$

$$
\varsigma_{K, N}^{(t)}(d)=t\left(\frac{\mathbf{s}_{K}(t d / \sqrt{N-1})}{\mathbf{s}_{K}(d / \sqrt{N-1})}\right)^{N-1}
$$

if $N>1$ and $K \in \mathbb{R}$, and $\varsigma_{K, 1}^{(t)}(d)=t$ if $K \leq 0$.

Now we can introduce the $\operatorname{MCP}(K, N)$ condition as defined by Ohta [20].

Definition 2.1. For $K \in \mathbb{R}, N>1$, or $K \leq 0$ and $N=1$ a metric measure space $(X, \mathrm{~d}, \mathfrak{m})$ is said to satisfy the $(K, N)$-measure contraction property, $\operatorname{MCP}(K, N)$ for short, if for every point $x \in X$ and a measurable set $A \subset X$ with $0<\mathfrak{m}(A)<\infty$ (and $A \subset$ $B(x, \pi \sqrt{(N-1) / K})$ is $K>0)$ there exists a probability measure $\boldsymbol{\pi}$ on $\mathrm{Geo}(X)$ such that

(1) We have $\left(e_{0}\right)_{\sharp} \boldsymbol{\pi}=\delta_{x}$ and $\left(e_{1}\right)_{\sharp} \boldsymbol{\pi}=\left.\mathfrak{m}(A)^{-1} \mathfrak{m}\right|_{A}$;

(2) For $t \in[0,1]$,

$$
\mathrm{d} \mathfrak{m} \geq\left(e_{t}\right)_{\sharp}\left(\varsigma_{K, N}^{(t)}(l(\gamma)) \mathfrak{m}(A) \mathrm{d} \boldsymbol{\pi}(\gamma)\right)
$$

holds as measures on $X$.

Let us then briefly give the connection of the $\operatorname{MCP}(K, N)$ condition to other similar curvature-dimension bounds. The $\operatorname{CD}(K, N)$ conditions by Sturm $[28,29]$ and Lott and Villani [18] use the same weights $\varsigma_{K, N}^{(t)}$ as $\operatorname{MCP}(K, N)$ but require a convexity type inequality between any two probability measures along some optimal transport geodesic. As shown by the second named author in $[25], \operatorname{CD}(K, N)$ implies $\operatorname{MCP}(K, N)$. There is also a more strict version of $\operatorname{MCP}(K, N)$ by Sturm [29]. He required the choice of geodesics be given by a Markov kernel. It is not known if the $\operatorname{CD}(K, N)$ conditions imply this more strict version of $\operatorname{MCP}(K, N)$. In this paper we will work with the definition of Ohta.

Changing slightly the weights $\varsigma_{K, N}^{(t)}$ to a more PDE-friendly versions give rise to so-called reduced definitions that usually carry a ' $*$ ' in their abbreviation. The first set of reduced definitions, $C^{*}(K, N)$ were considered by Bacher and Sturm in [5]. In nonbranching spaces it was shown by Cavalletti and Sturm [7] that $\mathrm{CD}^{*}(K, N)$ actually implies $\operatorname{MCP}(K, N)$. Recently Cavalletti has shown [6] that in nonbranching spaces $\operatorname{CD}^{*}(K, N)$ gives the required convexity-type inequalities for the $\mathrm{CD}(K, N)$ condition for a much larger class of transports. It is still open if the $\mathrm{CD}^{*}(K, N)$ really self-improves to $\mathrm{CD}(K, N)$.

The most recent additions to the set of definitions are the Riemannian Ricci curvature lower bounds $[2,1,10,3]$. These definitions start from the reduced weights and are therefore denoted by $\operatorname{RCD}^{*}(K, N)$. As the word 'Riemannian' in the name of the condition suggests, the $\operatorname{RCD}^{*}(K, N)$ condition rules out nonRiemannian Finsler structures that the $\mathrm{CD}^{*}(K, N)$ condition includes. As was noted in [12] the $\operatorname{RCD}^{*}(K, N)$ conditions imply $\operatorname{MCP}(K, N)$ without the nonbranching assumption that was assumed in [7]. As in the case of $\operatorname{CD}(K, N)$, it is not known if $\operatorname{RCD}^{*}(K, N)$ self-improves to $\operatorname{RCD}(K, N)$.

There are also other curvature-dimension conditions besides the ones mentioned so far. One interesting notion is the coarse Ricci curvature by Ollivier [23]. Because by [20] we know that $\operatorname{MCP}(K, N)$ spaces satisfy a Bishop-Gromov volume comparison theorem, the 


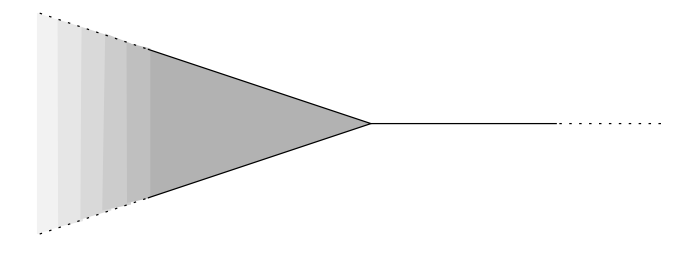

Figure 1. The space $(X, \mathrm{~d}, \mathfrak{m})$ satisfying $\operatorname{MCP}(0,3)$.

$\operatorname{MCP}(K, N)$ spaces (and in particular our examples) have a lower bound on the coarse Ricci curvature associated to the $r$-step random walk, see [16].

\section{ExAmples of $\operatorname{MCP}(K, N)$ SPACES With NON-CONStANT DimEnsion}

We now turn to the examples stated in Theorems 1.2 and 1.3. They are both constructed as closed connected subsets of $\mathbb{R}^{2}$ equipped with the distance $\mathrm{d}$ coming from the $l^{\infty}$-norm, i.e.

$$
\mathrm{d}\left(\left(x_{1}, y_{1}\right),\left(x_{2}, y_{2}\right)\right)=\sup \left(\left|x_{1}-x_{2}\right|,\left|y_{1}-y_{2}\right|\right) \quad \text { for all }\left(x_{1}, y_{1}\right),\left(x_{2}, y_{2}\right) \in \mathbb{R}^{2} .
$$

The first example we present here is a space satisfying $\operatorname{MCP}(0,3)$. It is a tangent space of the second space satisfying $\operatorname{MCP}(2,3)$. Therefore, instead of proving the $\operatorname{MCP}(0,3)$ property, we could refer to this fact. (See Section 3.3 for more discussion.) We will still prove the $\operatorname{MCP}(0,3)$ property in the first example because it is simpler and makes it easier to understand the second example.

In checking the examples we will use the convention that we are transporting from a dirac mass at $(\tilde{x}, \tilde{y})$ to a measure uniformly distributed on a set $A$.

3.1. Failure of topological splitting. Let us start with the easier example of Theorem 1.3 of a space $(X, \mathrm{~d}, \mathfrak{m})$ satisfying $\operatorname{MCP}(0,3)$ containing an isometric embedding of the euclidean real-line. As a subset of $\mathbb{R}^{2}$ the space $X$ is defined as

$$
\left\{(x, y) \in \mathbb{R}^{2}: x \leq-3|y| \leq 0\right\} \cup\left(\mathbb{R}_{+} \times\{0\}\right),
$$

see Figure 1 for an illustration.

The distance $\mathrm{d}$ on the space is the $l^{\infty}$-distance (3.1). The measure $\mathfrak{m}$ restricted to the halfinfinite line segment $L=\left(\mathbb{R}_{+} \times\{0\}\right)$ is $\left.\mathcal{H}^{1}\right|_{L}$, where $\mathcal{H}^{1}$ is the one-dimensional Hausdorff

measure. On the cone $C=\left\{(x, y) \in \mathbb{R}^{2}: x \leq-3|y| \leq 0\right\}$ we define $\mathfrak{m}$ as a weighted Lebesgue measure via

$$
\mathrm{d} \mathfrak{m}=\frac{3}{-2 x} \mathrm{~d} \mathcal{L}_{2}
$$

In other words, the projection of $\mathfrak{m}$ to the first coordinate is the Lebesgue measure $\mathcal{L}_{1}$ and the measure $\mathfrak{m}$ is equally distributed in $X \cap(\{x\} \times \mathbb{R})$ for all $x \in \mathbb{R}$.

Let us then check that the space $(X, \mathrm{~d}, \mathfrak{m})$ satisfies $\operatorname{MCP}(0,3)$. We will always transport measure linearly in the horizontal direction. Hence it is sufficient to check $\operatorname{MCP}(0,3)$ separately for sets $A_{\mathbf{x}}=\{(\mathbf{x}, y) \in A: y \in \mathbb{R}\}$ with $\mathbf{x} \in \mathbb{R}$. There are several cases to check depending on the position of the measure $\mu_{0}=\delta_{(\tilde{x}, \tilde{y})}$ and the coordinate $\mathbf{x} \in \mathbb{R}$. 

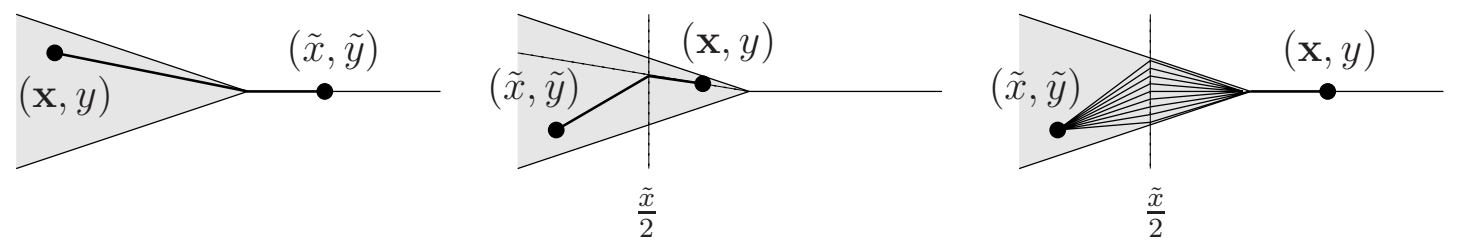

Figure 2. The cases where the selection of geodesics is not euclidean.

If $\mathbf{x}, \tilde{x}>0$, we are on the one-dimensional part and we have a unique geodesics from $(\tilde{x}, \tilde{y})$ to $(\mathbf{x}, y)$ and along such geodesics we have

$$
\frac{\mathrm{d} \mu_{t}}{\mathrm{~d} \mathfrak{m}}=\frac{1}{t} \frac{\mathrm{d} \mu_{1}}{\mathrm{~d} \mathfrak{m}} \leq \frac{1}{t^{3}} \frac{\mathrm{d} \mu_{1}}{\mathrm{~d} \mathfrak{m}} \quad \text { for all } t \in(0,1] .
$$

If $\mathbf{x}<0<\tilde{x}$, we can transport using a set of geodesics such that each image of a geodesic is a subset of a set of the form

$$
\{(x, y): y=a x, x \leq 0\} \cup L \quad \text { where }|a| \leq \frac{2}{3},
$$

in other words, we first transport along a euclidean geodesic to the origin and then continue along $L$. See the first case in Figure 2. Then the induced optimal transport satisfies

$$
\frac{\mathrm{d} \mu_{t}}{\mathrm{~d} \mathfrak{m}} \leq \frac{1}{t} \frac{\mathrm{d} \mu_{1}}{\mathrm{~d} \mathfrak{m}} \leq \frac{1}{t^{3}} \frac{\mathrm{d} \mu_{1}}{\mathrm{~d} \mathfrak{m}} \quad \text { for all } t \in(0,1] .
$$

Compared to the previous case the first equality has now change to an inequality due to the fact that the density might drop when we pass through the origin.

Next we have to check the different cases when $\tilde{x}<0$. Let us first look at the easier case where $\mathbf{x}<\tilde{x}$. In this case we can again use euclidean geodesics. Since the density of the reference measure $\mathfrak{m}$ with respect to $\mathcal{L}_{2}$ is increasing in $C$ as $x$ increases, we have the estimate

$$
\frac{\mathrm{d} \mu_{t}}{\mathrm{~d} \mathfrak{m}} \leq \frac{1}{t^{2}} \frac{\mathrm{d} \mu_{1}}{\mathrm{~d} \mathfrak{m}} \leq \frac{1}{t^{3}} \frac{\mathrm{d} \mu_{1}}{\mathrm{~d} \mathfrak{m}} \quad \text { for all } t \in(0,1]
$$

The final case is when $\tilde{x}<\mathbf{x}$. In this case we still have three subcases to select the geodesic(s) from $(\tilde{x}, \tilde{y})$ to a point $(\mathbf{x}, y)$. The division to subcases at $\frac{\tilde{x}}{2}$ is due to the fact that all geodesics from $(\tilde{x}, \tilde{y})$ to $\left(\left\{\frac{\tilde{x}}{2}\right\} \times \mathbb{R}\right) \cap C$ have the same length.

In the subcase $\tilde{x}<\mathbf{x} \leq \tilde{x} / 2$ we simply take the euclidean geodesic. In the subcase $\tilde{x} / 2<\mathbf{x}<0$ we take a geodesic that first goes along the euclidean geodesic from $(\tilde{x}, \tilde{y})$ to $\left(\frac{\tilde{x}}{2}, \frac{\tilde{x} y}{2 \mathrm{x}}\right)$ and then along the euclidean geodesic from $\left(\frac{\tilde{x}}{2}, \frac{\tilde{x} y}{2 \mathbf{x}}\right)$ to $(\mathbf{x}, y)$. See the second case in Figure 2. The third subcase is when $\mathbf{x}>0$. In this subcase we first spread the measure uniformly from $(\tilde{x}, \tilde{y})$ to $\{\tilde{x} / 2\} \times[-\tilde{x} / 6,-\tilde{x} / 6]$, then contract along the euclidean geodesics to the origin and then move to $(\mathbf{x}, y)$ along the euclidean geodesic. See the third case in Figure 2. Now in the case $\tilde{x}<\mathbf{x} \leq \tilde{x} / 2$ we have

$$
\frac{\mathrm{d} \mu_{t}}{\mathrm{~d} \mathfrak{m}}=\frac{1+\frac{2(\tilde{x}-\mathbf{x})}{\tilde{x}}(1-t)}{t^{2}} \frac{\mathrm{d} \mu_{1}}{\mathrm{~d} \mathfrak{m}} \leq \frac{2-t}{t^{2}} \frac{\mathrm{d} \mu_{1}}{\mathrm{~d} \mathfrak{m}} \leq \frac{1}{t^{3}} \frac{\mathrm{d} \mu_{1}}{\mathrm{~d} \mathfrak{m}} \quad \text { for all } t \in(0,1] .
$$


In the case $\tilde{x} / 2<\mathbf{x}$ we have

and

$$
\frac{\mathrm{d} \mu_{t}}{\mathrm{~d} \mathfrak{m}}=\frac{1}{t} \frac{\mathrm{d} \mu_{1}}{\mathrm{~d} \mathfrak{m}} \leq \frac{1}{t^{3}} \frac{\mathrm{d} \mu_{1}}{\mathrm{~d} \mathfrak{m}} \quad \text { for all } t \in[\tilde{x} /(2 \tilde{x}-2 \mathbf{x}), 1]
$$

$$
\frac{\mathrm{d} \mu_{t}}{\mathrm{~d} \mathfrak{m}}=\frac{2-\frac{2(\tilde{x}-\mathbf{x})}{\tilde{x}} t}{\left(\frac{2(\tilde{x}-\mathbf{x})}{\tilde{x}} t\right)^{2}} \frac{\mathrm{d} \mu_{1}}{\mathrm{~d} \mathfrak{m}} \leq \frac{2-t}{t^{2}} \frac{\mathrm{d} \mu_{1}}{\mathrm{~d} \mathfrak{m}} \leq \frac{1}{t^{3}} \frac{\mathrm{d} \mu_{1}}{\mathrm{~d} \mathfrak{m}} \quad \text { for all } t \in(0, \tilde{x} /(2 \tilde{x}-2 \mathbf{x})] .
$$

All cases now being checked we have shown that $(X, \mathrm{~d}, \mathfrak{m})$ satisfies $\operatorname{MCP}(0,3)$.

Remark 3.1. One can check that the space also satisfies the convexity inequality of the $\mathrm{CD}(0, N)$ condition for many transports. This is clearly the case for measures transported inside L. For transports between $C$ and $L$ this is less trivial, but still true. However, for transports in $C$ the $\mathrm{CD}(0, N)$ condition (in fact $\operatorname{CD}(K, \infty)$ for any $K \in \mathbb{R}$ ) fails. The problematic transports are the ones going in the diagonal direction where one is forced to use euclidean geodesics. Here the density change of the reference measure $\mathfrak{m}$ with respect to $\mathcal{L}_{2}$ destroys the $\mathrm{CD}(0, N)$ property.

It is not clear if the above example satisfies the $\operatorname{MCP}(0,3)$ condition as defined by Sturm [29]. In Sturm's definition one should be able to find a distribution of geodesics between any two points so that the $\operatorname{MCP}(K, N)$ condition is satisfied along the geodesics given by the distributions. The key point in the definition is that the distribution is symmetric.

3.2. Failure of topological maximal diameter theorem. The space $(Y, \mathrm{~d}, \mathfrak{n})$ we describe here with the maximal diameter $\pi$ is similar to our previous example. As a subset of $\mathbb{R}^{2}$ the space $Y$ is defined as

$$
Y=D \cup L
$$

where

$$
D=\left\{(x, y) \in \mathbb{R}^{2}: 9|y| \leq 1 / 4-|x|\right\} \quad \text { and } \quad L=([-\pi / 2,-1 / 4] \cup[1 / 4, \pi / 2]) \times\{0\} .
$$

See Figure 3 for an illustration. Again the distance $\mathrm{d}$ is the $l^{\infty}$-distance (3.1). The measure is similar to the measure $\mathfrak{m}$, but this time the projection of the measure to the interval $[-\pi / 2, \pi / 2]$ is of the form $\mathrm{d} \mathfrak{n}=\cos ^{2}(x) \mathrm{d} \mathcal{L}_{1}$. Again we define the measure in such a way that the density is constant on $(\{x\} \times \mathbb{R}) \cap Y$ for all $x \in[-\pi / 2, \pi / 2]$. The projected space $\left(\mathrm{p}_{1} Y, \mathrm{~d}_{e},\left(\mathrm{p}_{1}\right)_{\sharp} \mathfrak{n}\right)$ clearly satisfies $\mathrm{CD}(2,3)$. It is not that surprising that $(Y, \mathrm{~d}, \mathfrak{n})$ satisfies $\operatorname{MCP}(2,3)$ : the projection deals with the long distances and on the other hand it is already known that sufficiently small balls in $\mathbb{R}^{n}$ with the $l^{\infty}$ norm satisfy $\operatorname{MCP}(n, n+1)$, see [29]. The transition from the one-dimensional part to the two-dimensional part can then be carried out similarly as in the previous example.

Nevertheless, let us check that the space $(Y, \mathfrak{d}, \mathfrak{n})$ really satisfies $\operatorname{MCP}(2,3)$. Let $A \subset Y$ with $\mathfrak{n}(A)>0$ and $(\tilde{x}, \tilde{y}) \in Y$. We again transport linearly in the horizontal direction and so it suffices to check that for all $\mathbf{x} \in[-\pi / 2, \pi / 2]$ we can select geodesics $\gamma$ from $(\tilde{x}, \tilde{y})$ to $(\{\mathbf{x}\} \times \mathbb{R}) \cap A$ such that

$$
\frac{\mathrm{d} \mu_{t}}{\mathrm{~d} \mathfrak{n}}\left(\gamma_{t}\right) \leq \frac{\sin ^{2}(l(\gamma))}{t \sin ^{2}(t l(\gamma))} \frac{\mathrm{d} \mu_{1}}{\mathrm{~d} \mathfrak{n}}\left(\gamma_{1}\right) \quad \text { for all } t \in(0,1]
$$




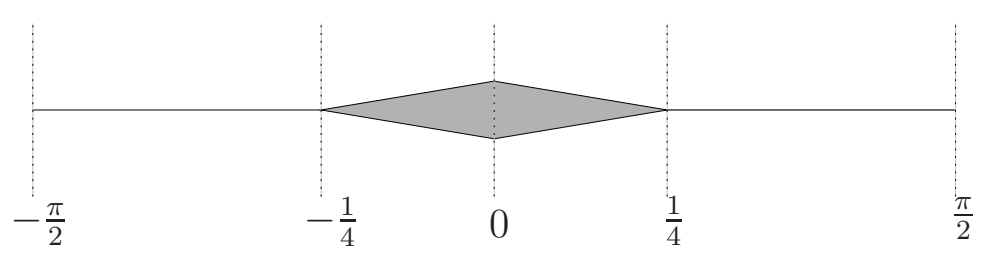

Figure 3. The space $(Y, \mathrm{~d}, \mathfrak{n})$ satisfying $\operatorname{MCP}(2,3)$.

We again consider different cases separately depending on the position of $(\tilde{x}, \tilde{y})$ and $\mathbf{x}$. First of all, if $1 / 4 \leq|\mathbf{x}|,|\tilde{x}| \leq \pi / 2$ we can take the measures $\mu_{t}$ to be uniformly distributed on the slices $(\{x\} \times \mathbb{R}) \cap Y$. Since the space $\left(\mathrm{p}_{1} Y, \mathrm{~d}_{e},\left(\mathrm{p}_{1}\right)_{\sharp} \mathfrak{n}\right)$ satisfies $\operatorname{CD}(2,3)$, we have the desired inequality (3.2) in this case.

The second case is when $|\mathbf{x}| \leq 1 / 4$ and $1 / 4 \leq|\tilde{x}| \leq \pi / 2$. In this case we take the geodesics along which we transport to be such that their images are subsets of sets of the type

$$
L \cup\left\{(x, y) \in \mathbb{R}^{2}: c y=1 / 4-|x| \geq 0\right\}, \quad \text { with }|c| \geq 9 .
$$

Because inside $D$ we are changing the density as if we were transporting in $\left(\mathrm{p}_{1} Y, \mathrm{~d}_{e},\left(\mathrm{p}_{1}\right)_{\sharp} \mathfrak{n}\right)$ and the density relative to this transport only drops when we leave $D$, again the fact that we have the inequality (3.2) follows from the observation that the space $\left(\mathrm{p}_{1} Y, \mathrm{~d}_{e},\left(\mathrm{p}_{1}\right)_{\sharp} \mathfrak{n}\right)$ satisfies $\mathrm{CD}(2,3)$.

The final case to check is when $|\tilde{x}| \leq 1 / 4$. By symmetry we may assume that $\tilde{x} \leq \mathbf{x}$. As in the previous example we define the chosen geodesics from $(\tilde{x}, \tilde{y})$ to a point $(\mathbf{x}, y) \in A$ in three subcases. This time the division to subcases is driven by the fact that the distances from $(\tilde{x}, \tilde{y})$ to all the points in $(\{(1 / 4+4 \tilde{x}) / 5\} \times \mathbb{R}) \cap D$ are equal, meaning that beyond that distance we can transport along vertically uniformly distributed measures.

Let us list the subcases. First, if $\tilde{x}<\mathrm{x} \leq(1 / 4+4 \tilde{x}) / 5$, we take the euclidean geodesics. In the subcase $(1 / 4+4 \tilde{x}) / 5<\mathbf{x}<1 / 4$ we take a geodesic that first goes along the euclidean geodesic that goes via the point $(1 / 4,0)$ from $(\mathbf{x}, y)$ to $\left(\max \{0,(1 / 4+4 \tilde{x}) / 5\}, y^{\prime}\right)$ and then along the euclidean geodesic from $\left(\max \{0,(1 / 4+4 \tilde{x}) / 5\}, y^{\prime}\right)$ to $(\tilde{x}, \tilde{y})$. The third subcase is when $\mathbf{x}>1 / 4$. In this subcase we first transport the mass along the euclidean geodesic to the point $(1 / 4,0)$, then spread the measure uniformly to $(\{\max \{0,(1 / 4+4 \tilde{x}) / 5\}\} \times \mathbb{R}) \cap D$ along euclidean geodesics and finally contract it to $(\tilde{x}, \tilde{y})$ along euclidean geodesics.

Let us check the density in the different subcases. To deal with the first subcase $\tilde{x}<$ $\mathbf{x} \leq(1 / 4+4 \tilde{x}) / 5$ we observe that $h_{t} \leq(5 / 4-t / 4) h_{1}$ for the vertical height $h_{t}$ of $D$ at $\gamma_{t}$, where $\gamma$ is a geodesic from $(\tilde{x}, \tilde{y})$ to $(\mathbf{x}, y)$. From this (and the estimates below) we have that

$$
\frac{\mathrm{d} \mu_{t}}{\mathrm{~d} \mathfrak{n}} \leq \frac{h_{t}}{t^{2} h_{1}} \frac{\mathrm{d} \mu_{1}}{\mathrm{~d} \mathfrak{n}} \leq \frac{(5 / 4-t / 4)}{t^{2}} \frac{\mathrm{d} \mu_{1}}{\mathrm{~d} \mathfrak{n}} \leq \frac{\sin ^{2}(l)}{t \sin ^{2}(t l)} \frac{\mathrm{d} \mu_{1}}{\mathrm{~d} \mathfrak{n}}
$$

for all $t \in(0,1]$ and $l \in(0,1 / 2]$ and so (3.2) follows. (To see the third inequality in (3.3) define $f_{l}(t):=\left(\frac{5}{4}-\frac{t}{4}\right) \sin ^{2}(t l)-t \sin ^{2}(l)$ and notice that $f_{l}(t) \leq 0$ for all $t \in[0,1]$ and $l \in(0,1 / 2]$ since $f_{l}(0)=f_{l}(1)=0$ and $f_{l}^{\prime \prime}(t)=-\frac{l}{2} \sin (2 t l)+(5-t) \frac{l^{2}}{2} \cos (2 t l) \geq 0$ for all $t \in[0,1]$ and $l \in(0,1 / 2]$. 
In the second and third subcases $(1 / 4+4 \tilde{x}) / 5<\mathbf{x}$, until we hit $\left(\max \{0,(1 / 4+4 \tilde{x}) / 5\}, y^{\prime}\right)$ we have $(3.2)$ by the projection as before. After $\left(\max \{0,(1 / 4+4 \tilde{x}) / 5\}, y^{\prime}\right)$ we have $(3.2)$ by the estimate $(3.3)$ for $l \in\left(0, \frac{1}{2}\right]$. When $l>\frac{1}{2}$ we can estimate the time $t_{0}$ when we hit $\left(\max \{0,(1 / 4+4 \tilde{x}) / 5\}, y^{\prime}\right)$ by

$$
t_{0} \leq \frac{1}{l}\left(\frac{\frac{1}{4}+4 \tilde{x}}{5}-\tilde{x}\right) \leq 2 \frac{\frac{1}{4}-\tilde{x}}{5} \leq \frac{1}{5} .
$$

In this case obtaining (3.2) is easy, since verifying (3.3) for $t \in\left[0, \frac{1}{5}\right]$ and $l \in\left(\frac{1}{2}, \frac{\pi}{2}+\frac{1}{4}\right)$ reduces to verifying that $f_{l}(t) \leq 0$ for such $t$ and $l$. This follows for example by estimating

$$
\left(\frac{5}{4}-\frac{t}{4}\right) \sin ^{2}(t l) \leq \frac{5}{4} \sin ^{2}(t l) \leq \frac{5}{4}(t l)^{2} \leq \frac{1}{4} t l^{2} \leq t \sin ^{2}(l)
$$

for $t \in\left[0, \frac{1}{5}\right]$ and $l \in\left(\frac{1}{2}, \frac{\pi}{2}+\frac{1}{4}\right)$. This completes the proof of the $\operatorname{MCP}(2,3)$ condition for our space $(Y, \mathrm{~d}, \mathfrak{n})$.

3.3. Final remarks. Both of our examples above had boundary points in the sense that there are geodesics that cannot be infinitely extended as local geodesics. It is natural to ask if one can modify the examples to have no boundary. By identifying the top and bottom boundaries in the two-dimensional parts of the spaces we should be able to get rid of most of the boundary points. However, even after the identification the point where the space changes from one-dimensional to two-dimensional will be a boundary point in the above sense. The nonextendable geodesics are the nonvertical geodesics in the two-dimensional side that are travelling more in the vertical direction than in the horizontal direction. When they reach the point where the dimension changes they cannot be extended to the one-dimensional part so that they are geodesic in a neighbourhood of the point where the dimension changes.

It is also interesting to consider the tangent spaces of the spaces $(X, \mathrm{~d}, \mathfrak{m})$ and $(Y, \mathrm{~d}, \mathfrak{n})$ in the sense of measured Gromov-Hausdorff limits of blow-ups. First observation is that the space $(X, \mathrm{~d}, \mathfrak{m})$ is the $\mathrm{mGH}$-tangent of $(Y, \mathrm{~d}, \mathfrak{n})$ at the points $(a, 0)$ and $(-a, 0)$. This way we could prove the fact that $(X, \mathrm{~d}, \mathfrak{m})$ satisfies $\operatorname{MCP}(0,3)$ by proving that any tangent of a $\operatorname{MCP}(K, 3)$ space does.

Notice that the tangents of $(X, \mathrm{~d}, \mathfrak{m})$ at points $X \backslash\{(0,0)\}$ and $(Y, \mathrm{~d}, \mathfrak{n})$ at points $Y \backslash$ $\{(1 / 4,0),(-1 / 4,0)\}$ are all convex subsets of normed spaces. On the other hand we know from [14] that some subRiemannian spaces like the Heisenberg group satisfy $\operatorname{MCP}(K, N)$. With only such nice examples at hand it is natural to ask what are the tangent spaces of $\operatorname{MCP}(K, N)$ spaces (almost everywhere) in general? This is also related to the uniqueness of the tangents, compare to the following result by Le Donne [17].

Theorem 3.2 (Le Donne). Let $(Z, \mathrm{~d}, \mu)$ be a doubling-measured geodesic metric space. Assume that for $\mu$-almost every $x \in Z$ the space $Z$ has only one tangent. Then for $\mu$ almost every point $x \in Z$ the tangent space is a Carnot group $\mathbf{G}$ endowed with a subFinsler left-invariant metric with the first layer of the Lie algebra of $\mathbf{G}$ as horizontal distribution. 


\section{REFERENCES}

[1] L. Ambrosio, N. Gigli, A. Mondino and T. Rajala, Riemannian Ricci curvature lower bounds in metric measure spaces with $\sigma$-finite measure, arxiv:1207.4924, (2012), to appear on Trans. Amer. Math. Soc.

[2] L. Ambrosio, N. Gigli and G. Savaré, Metric measure spaces with Riemannian Ricci curvature bounded from below, Preprint, arXiv:1109.0222, (2011), to appear on Duke Math. J.

[3] L. Ambrosio, A. Mondino, and G. Savaré, Nonlinear diffusion equations and curvature conditions in metric measure spaces, In progress, (2013).

[4] L. Ambrosio, A. Mondino, and G. Savaré, On the Bakry-Émery condition, the gradient estimates and the Local-to-Global property of $R C D^{*}(K, N)$ metric measure spaces Preprint, arXiv:1309.4664, (2013).

[5] K. Bacher and K.-T. Sturm, Localization and tensorization properties of the curvature-dimension condition for metric measure spaces, J. Funct. Anal., 259 (2010), 28-56.

[6] F. Cavalletti, Decomposition of geodesics in the Wasserstein space and the globalization property, arXiv:1209.5909, (2012), to appear on Geom. Funct. Anal.

[7] F. Cavalletti and K.-T. Sturm, Local curvature-dimension condition implies measure-contraction property, J. Funct. Anal., 262 (2012), 5110-5127.

[8] J. Cheeger and D. Gromoll, The splitting theorem for manifolds of nonnegative Ricci curvature, J. Differential Geometry, 6 (1971/72), 119-128.

[9] Y. Cheng, Eigenvalue comparison theorems and its geometric applications, Math. Z. 143 (1975), 289297.

[10] M. Erbar, K. Kuwada and K.-T. Sturm, On the equivalence of the entropic curvature-dimension condition and Bochner's inequality on metric measure spaces, Preprint, arXiv:1303.4382, (2013).

[11] N. Gigli, The splitting theorem in non-smooth context, Preprint, arXiv:1302.5555, (2013).

[12] N. Gigli, A. Mondino and T. Rajala, Euclidean spaces as weak tangents of infinitesimally Hilbertian metric spaces with Ricci curvature bounded below, arXiv:1304.5359, (2013), to appear in J. Reine Angew. Math.

[13] N. Gigli, T. Rajala and K.-T. Sturm, Optimal maps and exponentiation on finite dimensional spaces with Ricci curvature bounded from below, Preprint, arXiv:1305.4849, (2013).

[14] N. Juillet, Geometric inequalities and generalized Ricci bounds in the Heisenberg group, Int. Math. Res. Notices 2009 (2009), 2347-2373.

[15] C. Ketterer, Cones over metric measure spaces and the maximal diameter theorem, Preprint, arXiv:1311.1307, (2013).

[16] Y. Kitabeppu, Lower bound of coarse Ricci curvature on metric measure spaces and eigenvalues of Laplacian, to appear on Geom. Dedicata, doi:10.1007/s10711-013-9844-3

[17] E. Le Donne, Metric spaces with unique tangents, Ann. Acad. Sci. Fenn. Math. 36 (2011), no. 2, 683-694.

[18] J. Lott and C. Villani, Ricci curvature for metric-measure spaces via optimal transport, Ann. of Math. 169 (2009), no. 3, 903-991.

[19] S.-i. Ohta, Examples of spaces with branching geodesics satisfying the curvature-dimension condition, to appear on Bull. Lond. Math. Soc.

[20] S.-i. Ohta, On the measure contraction property of metric measure spaces, Comment. Math. Helv. 82 (2007), 805-828.

[21] S.-i. Ohta, Products, cones, and suspensions of spaces with the measure contraction property, J. Lond. Math. Soc. (2) 76 (2007), 225-236.

[22] S.-i. Ohta, Splitting theorems for Finsler manifolds of nonnegative Ricci curvature, to appear in J. Reine Angew. Math.

[23] Y. Ollivier, Ricci curvature of Markov chains on metric spaces, J. Funct. Anal. 256 (2009), 810-864.

[24] T. Rajala, Failure of the local-to-global property for $C D(K, N)$ spaces, arXiv:1305.6436, (2013), to appear in Ann. Sc. Norm. Super. Pisa Cl. Sci. 
[25] T. Rajala, Interpolated measures with bounded density in metric spaces satisfying the curvaturedimension conditions of Sturm, J. Funct. Anal., 263 (2012), no. 4, 896-924.

[26] T. Rajala, Local Poincaré inequalities from stable curvature conditions on metric spaces, Calc. Var. Partial Differential Equations, 44 (2012), no. 3-4, 477-494.

[27] T. Rajala and K.-T. Sturm, Non-branching geodesics and optimal maps in strong $C D(K, \infty)$-spaces, arXiv:1207.6754, (2012), to appear in Calc. Var. Partial Differential Equations.

[28] K.-T. Sturm, On the geometry of metric measure spaces. I, Acta Math. 196 (2006), no. 1, 65-131.

[29] K.-T. Sturm, On the geometry of metric measure spaces. II, Acta Math. 196 (2006), no. 1, 133-177.

Institut für Angewandte Mathematik, Universität Bonn, Endenicher Allee 60, D-53115 Bonn, Germany

E-mail address: ketterer@iam.uni-bonn.de

Department of Mathematics and Statistics, P.O.Box 35 (MaD), FI-40014 University of JYVÄSKYLÄ, FINLAND

E-mail address: tapio.m.rajala@jyu.fi 